\title{
De la enseñanza de los conocimientos teóricos en medicina
}

\author{
F. Baquero
}

\section{¿Es la medicina una ciencia?}

Sólo la ciencia requiere conocimientos teóricos; sólo si la medicina es una ciencia y el médico un científico, la formación teórica es necesaria. Médicos de ignorancia y simplicidad acreditada, pero de buena voluntad y con buen concepto de sí mismos, podrían poner en duda si el conocimiento científico es necesario para ejercer la medicina, y podrían proponer que la ésta es meramente un conjunto de prácticas útiles y contrastadas que, en todo caso, se nutre de las 'ciencias verdaderas'. Sin embargo, no debería ser posible siquiera discutir que la medicina científica, que trata de comprender la razón de los cambios en la fisiología de un ser biológico particular, el hombre, que son atribuibles a la enfermedad, y de prevenirlos o curarlos, es claramente una parte de las ciencias de la vida, una parte de la biología. Es interesante que la pregunta ¿es la medicina una ciencia? también se haya planteado durante mucho tiempo de la propia ciencia-madre, de la biología. ¿Es la biología una ciencia, como las verdaderas ciencias, sometida a leyes y con un carácter estrictamente cuantitativo, como la física o la química?, ¿o bien, es más una 'ciencia provinciana', ya que carece de universalidad, y sus descubrimientos pueden reducirse, en último término, a las leyes de la física y la química?

\section{La medicina en la perspectiva utilitaria}

La perspectiva utilitaria es la que permite plantearse esta cuestión crítica: ¿para qué hacen falta los conocimientos teóricos en medicina? Una detección, incluso automatizada, de signos y síntomas podría llevar a un diagnóstico y un tratamiento, e incluso realizar pronósticos, sin necesidad de conocer los procesos básicos de la etiología, la fisiopatología o la patogenia. Incluso en nuestros días, el enfermo podría asemejarse para algunos médicos utilitaristas a una caja negra (se desconocen, por innecesarios, los mecanismos que contiene), que 'exhibe' hacia el exterior un conjunto de signos y síntomas, detectables fácilmente a través de la tecnología médica, incluso sin participación del médico, que producen conjuntos derivados de datos que adecuadamente tratados por vía informática y de forma objetiva (incluso de forma 'basada en la evidencia') dan lugar a recomendaciones terapéuticas precisas (también 'basadas en la evidencia') y expectativas pronósticas que deberían resolver la mayor parte de los casos de enfermedad. Por supuesto, existe aquí una cuestión de petición de principio: sin que alguien 'haga ciencia' en la medicina, esos diagnósticos y tratamientos 'automatizados', accesibles a médicos no científicos, no se hubiesen desarrollado ni validado nunca. Esta reflexión plantea una tercera vía: habría que hacer coexistir médicos 'prácticos', en una perspectiva casi 'paramédica', y médicos 'científicos', responsables del desarrollo de la medicina. Esta distinción es académicamente perversa. La medicina, toda la medicina, como ciencia, debe estar en todo médico. La ciencia no puede sustituirse por el sentido común de los médicos prácticos. Fue un médico, Thomas Henry Huxley, amigo de Darwin (1825-1892), el que planteó que la ciencia (y debía referirse a
Profesor Ramón y Cajal de Investigación. Fundación de Investigación Biomédica del Hospital Ramón y Cajal. Servicio de Microbiología. Hospital Universitario Ramón y Cajal.

Unidad Asociada de Investigación RYC-CNB (CSIC). Madrid, España.

E-mail

baquero@bitmailer.net

Agradecimientos A la Dra. Rosario Artigao, que me ha enseñado cómo se debe aliar el conocimiento científico con la más profundamente humanizada práctica médica. 
la medicina) 'no es más que sentido común entrenado y organizado'. Como replicó mucho más tarde Ernst Mayr, el Darwin del siglo xx (19052005), que pasó desde los estudios médicos a los estrictamente biológicos, por desgracia, esto no es cierto, sino al contrario, frecuentemente la ciencia corrige el sentido común, e incluso 'se podría decir que la ciencia consiste en confirmar o refutar el sentido común'.

\section{De los conocimientos científicos}

Los conocimientos teóricos básicos, los conocimientos científicos, son aquellos que identifican los elementos y explican los procesos que determinan la historia natural de la salud y enfermedad. Para el médico, estos conocimientos posibilitan:

- Un lenguaje científico común.

- Una reflexión autónoma y crítica.

- La confianza necesaria para la toma de decisiones clínicas ('entender para actuar').

- El avance científico, tanto a nivel personal ('la ciencia personal') como colectivo.

En otras palabras, los conocimientos científicos permiten 'explicar' (y explicarse) el enfermo, y esto es la forma de ejercer la ciencia.

En efecto, la ciencia es la 'explicación' (como desplegar un papel doblado para observar lo que hay dentro) de los fenómenos naturales. Sólo los conocimientos teóricos facilitan la explicación, y el médico debe saber, incluso estar impregnado en la convicción, de que no todo enfermo, o quizá ningún enfermo, puede ser siempre totalmente 'explicado' de acuerdo con patrones y reglas. Todo enfermo se ofrece al médico como un problema de alta complejidad, excepto si el médico se refugia de éste aplicando recetas genéricas. Pues bien, la 'complejidad' de cada enfermo, y por tanto la comprensión del mismo, sólo puede abarcarse a través de síntesis individuales, que debe realizar el médico. La explicación (siquiera parcial) de la complejidad presente en cada enfermo implica una combinatoria sintética personal de elementos adquiridos con el conocimiento teórico. El intento de 'explicar' el enfermo es la raíz de cualquier medicina científica. Para ello, tanto a nivel general como en el nivel debido a
Tabla I. De los conocimientos teóricos necesarios para una medicina científica.

- Anatomía general

- Bioquímica y metabolismo

- Biología y genética molecular

- Fisiología y adaptación

- Genética general

- Inmunología general

- Microbiología e interacciones huésped-parásito

- Biología y biopatología celular y subcelular

- Biología y biopatología de órganos y tejidos

- Biología y biopatología de los grandes sistemas orgánicos

- Redes nosológicas

- Farmacología general

- Psicología y etología

- Patología general y semiología

- Métodos diagnósticos

- Epidemiología y ecología

- Biología evolutiva

- Bioestadística, bioinformática y modelización

- Información médica

- Método y crítica científica; ciencia y pseudociencia

- Modelos para ciencia translacional

- Ética médica y comunicación enfermo-paciente

- Historia de la ciencia médica

- Organización sanitaria y normas sociales en medicina

- Medicina y humanidades

cada especialidad médica, debe ofrecerse al estudiante de medicina un conjunto completo de conocimientos teóricos que agrupamos arbitrariamente en 25 tipos en la tabla.

\section{De la mentalidad investigadora en la práctica médica}

Cualquier médico debería reconocerse a sí mismo como un investigador en medicina, a no ser que su ignorancia o autosatisfacción (si es que ambas calidades pudiesen distinguirse) le hiciese creer que todo está resuelto, o, en todo caso, que él no tiene nada que ver con el progreso de la medicina. Sin embargo, el médico, que siempre se enfrenta a la complejidad de la enfermedad y que, lo crea o no, siempre se enfrenta a casos únicos, es el mejor situado para encender la cerilla de la investigación, que apunta a la dirección adecuada y da una pequeña luz inicial que puede desarrollarse más tarde por verdaderos equipos de investigación. 
Cuando el médico se hace preguntas como las siguientes: ¿es posible obtener de mi experiencia, con una muestra de enfermos, alguna conclusión general para todo este tipo de enfermos?, ¿se puede sacar de mi experiencia, con una muestra de enfermos, una pregunta general aplicable a estos enfermos?, ¿ estoy satisfecho de cómo se manejan este tipo de enfermos en general?, el médico que se plantea estas preguntas es un investigador, esto es, contribuye al progreso de la medicina.

Contribuir al progreso de la ciencia médica es una obligación de cada médico, y esta actividad necesaria es simplemente imposible sin conocimientos teóricos. Podemos realizar la siguiente secuencia lógica:

- Una verdadera ciencia es aquella que asegura su propio progreso, incrementando sus conocimientos.

- El progreso de los conocimientos depende de la investigación científica.

- La medicina tiene la responsabilidad de investigar para progresar, y si los médicos renuncian a esa actividad (p. ej., desdeñando la adquisición de conocimientos teóricos), la medicina dejaría se ser una ciencia y se convertiría en un conjunto acartonado de reglas de actuación.

\section{De la convergencia metodológica entre la práctica médica y la investigación}

Cualquier análisis teórico, es decir, científico, del enfermo (o de grupos coherentes de enfermos) evoluciona en torno a las mismas preguntas que constituyen la base de cualquier actividad investigadora. Estas preguntas se refieren, consecutivamente, a:

- La exploración (¿qué me llama la atención?).

- La explicación inicial (¿cómo podría explicarse?).

- La comprobación (¿es realmente así?).

- El establecimiento de principios causales generales (¿por qué?, ¿qué causas y condiciones han intervenido en la aparición o evolución de esta enfermedad?).

- El control (¿modifico algo si modifico las causas y condiciones?).
- La predicción (¿puedo prever su aparición si aparecen las causas y condiciones?).

Hacer medicina es pues trabajar con los mismos esquemas mentales que el investigador. En la medicina práctica cotidiana se puede dar un paso más, sistematizando la recolección de datos y la acumulación de conocimientos de base observacional o descriptiva a nivel personal o colectivo (grupos de médicos o colaboración con otros profesionales sanitarios).

Es un error considerar que la investigación médica es de carácter necesariamente experimental. La aplicación de conocimientos teóricos (explicativos) para sistematizar la mera observación, es también ciencia. Como dice Ernst Mayr, 'no hay motivo para sentirse avergonzado' por hacer ciencia observacional o descriptiva, $y$, en realidad, la genómica más avanzada no difiere de ello mucho de las descripciones clínicas. De hecho, la ciencia se ha definido como la 'organización y clasificación del conocimiento sobre la base de principios explicativos'.

\section{De los conocimientos teóricos en el entrenamiento del entendimiento médico}

Sir William Osler, en su discurso de apertura del Instituto Wistar de Anatomía y Biología, pronunciado en Filadelfia el 21 de mayo de 1894, y significativamente titulado La levadura de la ciencia, nos dice: 'Especialmente para el médico, una disciplina científica es un don incalculable que fermenta toda su vida.... $Y$ añade, con mayor énfasis, 'La perdición espera inevitablemente a la mente del médico que nunca ha sido plenamente inoculado con la levadura, que nunca ha captado las relaciones de la ciencia con su arte, y que no sabe nada, y quizá ni le importa, de sus limitaciones'. Así que existe una 'gran necesidad de hombres que sean pensadores además de trabajadores, hombres con ideas, que hayan bebido hasta el fondo la copa del vino astral.... Pero no sólo los conocimientos teóricos -el vino astralpermiten hacer medicina, sino, sobre todo, ejercitar la mente del médico, ya que, como hemos visto, no existe una separación formal entre la forma mental de hacer medicina y de hacer investigación. Osler insiste mucho en este punto: 
'La levadura de la ciencia proporciona a los hombres hábitos de precisión mental. Fortalece -para usar una expresión de Epicarno- los músculos y tendones del entendimiento'.

\section{Del placer de hacer medicina a la luz de los conocimientos teóricos}

La naturaleza premia con un intenso placer intelectual al médico y al investigador en general que realiza cualquier descubrimiento, aun con el más aparentemente insignificante. Como dice Stern: 'Percibir un hecho de la naturaleza que jamás había sido visto por un ojo o una mente humana, descubrir una nueva verdad en algún campo, revelar un acontecimiento de la historia pasada o identificar una relación oculta... El afortunado que haya vivido estas experiencias se recreará en ellas toda su vida.... ¿Porqué privar al médico de estos placeres, por una vez ligados a una acción indudablemente positiva para la sociedad? El peor castigo de la ignorancia es la privación de los placeres del conocimiento, pero estos placeres requieren la adquisición de conocimientos teóricos. Permitamos que se haga constantemente ciencia con la medicina, ciencia universal, comunitaria, desinteresada, basada en un sano escepticismo organizado. En palabras de uno de los mayores filósofos contemporáneos de la ciencia, Kart Popper: 'Después de la música y el arte, la ciencia es el mayor, el más bello y el más iluminado logro del espíritu humano. O, incluso, como dijo Herbert Spencer, en su famoso libro Education: 'El conocimiento científico es el más importante de todo tipo de conocimiento humano, para la autopreservación, para el mantenimiento de la vida y la salud, para la producción intelectual y artística, incluso para poseer la necesaria disciplina intelectual y moral que requiere la dignidad humana'. Sin conocimientos teóricos no hay espíritu.

\section{Bibliografía}

1. Mayr E. This is biology, the science of the living world. Cambridge: Harvard University Press; 1997.

2. Merton RK. A note on science and democracy. Journal of Legal and Political Sociology 1942; 1: 115-26.

3. Nagel E, Hawking D. The structure of science. Am J Phys 1961; 29: 716.

4. Spencer H. Education. London: Watts \& Co.; 1929.

5. Stern C. Thoughts on research. Science 1965; 148: 772. 